\title{
In Situ Existing Lead
}

National Cancer Institute

\section{Source}

National Cancer Institute. In Situ Existing Lead. NCI Thesaurus. Code C101239.

An insulated electrical conductor designed to connect to an electrical device for which the lead is in situ. (ACC) 